\title{
MULTI RESOLUTION LATTICE DISCRETE FOURIER TRANSFORM (MRL-DFT)
}

\author{
Fred L. Fontaine \\ Department of Electrical Engineering, Cooper Union, New York City, USA
}

\begin{abstract}
In many imaging applications, including sensor arrays, MRI and CT,data is often sampled on non-rectangular point sets with non-uniform density. Moreover, in image and video processing, a mix of non-rectangular sampling structures naturally arise. Multirate processing typically utilizes a normalized integer indexing scheme, which masks the true physical dimensions of the points. However, the spatial correlation of such signals often contains important information.This paper presents a theory of signals defined on regular discrete sets called lattices, and presents an associated form of a finite Fourier transform denoted here as multiresolution lattice discrete Fourier transform (MRL-DFT). Multirate processing techniques such as decimation, interpolation and polyphase representations are presented in a context which preserves the true spatial dimensions of the sampling structure.Moreover, the polyphase formulation enables systematic representation and processing for sampling patterns with variable spatial density, and provides a framework for developing generalized FFT and regridding algorithms.
\end{abstract}

\section{KEYWORDS}

Image Processing, Video, Medical Imaging, Multirate Signal Processing, Fourier Transform.

\section{INTRODUCTION}

A number of imaging applications involve data located on non-rectangular grids. Examples include tomographic schemes such as medical computerized tomography (CT) and certain synthetic aperture radar (SAR) systems, in which data is often acquired on a polar grid [1,2]. In magnetic resonance imaging (MRI), data is acquired in the two- or three-dimensional spatial frequency domain on sometimes quite complex patterns, such as the spiral shown in Figure 1. Different types of video signals have spatio-temporal data on different sampling structures, such as interlaced versus progressive. As illustrated with the spiral pattern, these irregular sampling patterns often have variable spatial density. Many common signal processing applications such as filtering and spectral analysis are difficult to accomplish, and conversion among sampling structures without introducing artifacts can be challenging [1,3].Data is often converted to a uniform rectangular grid, through an interpolation process called regridding, to facilitate processing, as in [4], but there may be significant artifacts caused by density mismatch.

Some methods have been developed for certain irregular structures as special cases, as in $[5,6,7]$, as well as for uniform but non-rectangular sampling patterns, called lattices, as in [3,8]. A normalized integer indexing scheme is typically used, which does not reflect true underlying geometric factors such as distance and direction. However, for many multidimensional signals, significant information is contained in the spatial correlation properties. For example, in [9], wireless network nodes are located on scattered points in a lattice and information regarding geometric location is vital for proper signal processing. In [10], a lattice based approach is used

Dhinaharan Nagamalai et al. (Eds) : ITCCMA, CSITY, AIFZ, NWCOM, SIGPRO - 2017

pp. 71- 90, 2017. (C) CS \& IT-CSCP 2017

DOI : $10.5121 /$ csit.2017.70706 
to analyse interference signals. In certain digital communication applications, as in $[11,12,13]$, signal sets are defined on lattices, and the geometric properties of the lattices are central to the development of receiver algorithms and performance analysis.

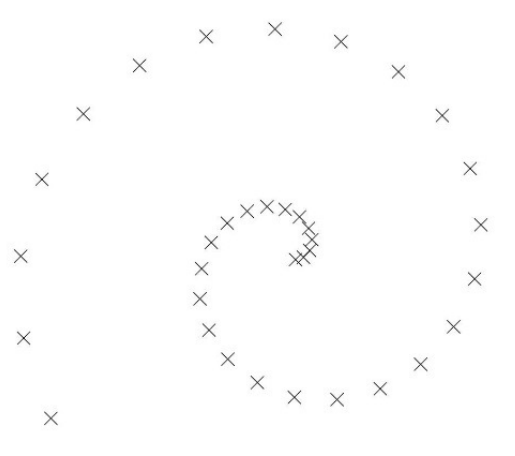

Figure 1: Sample points along a spiral.

Although conversion between different sampling structures is common, usually a single pattern containing various spatial sampling densities is not employed. This paper presents a systematic approach for dealing with multidimensional sampling patterns, including possibly with variable density. The results are formulated using physical coordinate systems for the spatial and frequency domains to facilitate development of algorithms such as filtering and interpolation (regridding) for sampling structure conversion. In particular, we study a finite Fourier transform suitable for this context, denoted here as the multiresolution lattice discrete Fourier transform (MRL-DFT). The MRL-DFT is used to guide the design of filters, sampling structure conversions, and polyphase structures, as well as the study of lattices to facilitate manipulating sampling patterns with variable densities.

We first describe lattices as sampling structures, viewed both in the standard normalized coordinates [3] and in physical coordinate systems, and develop an associated Fourier theory. We then discuss various signal processing operations and properties of lattice structures based on the MRL-DFT, and then provide a detailed example of how the methods developed here can facilitate various signal processing applications.

\section{LATTICES}

In this section, we introduce some notation and summarize basic definitions and results regarding lattices, and describe the topic under study in this paper.

\subsection{Notation and Definitions}

We consider complex valued signals whose domains are certain subsets of $D$-dimensional real space, $\mathcal{R}^{D}$. If $\mathcal{S} \subset \mathcal{R}^{D}, \vec{r} \in \mathcal{R}^{D}$ and $A$ is a $D \times D$ matrix, then $A \mathcal{S}+\vec{r}=\{A \vec{s}+\vec{r}$ : $\vec{s} \in \mathcal{S}\}$.If $\mathcal{S}$ is a finite set, $|\mathcal{S}|$ denotes the number of elements in $\mathcal{S}$.

We can associate vectors with column vectors, and in this form, the dot product (or inner product) of vectors $\mathcal{R}^{D}$ can be expressed as $\langle\vec{a}, \vec{b}\rangle=\vec{a} \cdot \vec{b}=a^{T} b$. In this paper, we will work with a non-radian frequency domain; for example, if the spatial vector $\vec{r}$ has units of meters, the frequency vector $\vec{f}$ has units of (meter) ${ }^{-1}$. This suggests the following notation:

$$
e(\alpha)=\exp (j 2 \pi \alpha)
$$


Note that $e(\alpha)=1$ iff $\alpha \in Z$. Then the Fourier transform of a signal $x(\vec{r})$ can be thought of as a representation of the signal as a superposition of sinewaves of the form $e(<\vec{f}, \vec{r}\rangle)$ for a suitable collection of frequency vectors $\vec{f}$.

Definition 1: A $D$-dimensional lattice $\mathcal{L} \subset \mathcal{R}^{D}$ is a set of the form:

$$
\mathcal{L}=V Z^{D}=\left\{\vec{r}=V \vec{n}: \vec{n} \in Z^{D}\right\}
$$

for some $D \times D$ nonsingular matrix $V$.

In this paper, all matrices we consider (e.g., lattice generators) are invertible $D \times D$ real. Given a lattice $\mathcal{L}$, the associated matrix $V$, called a generator of the lattice, is not unique. We denote a lattice generated by $V$ as $\mathcal{L}_{V}$. A signal $x(\vec{r})$ is said to have support on a lattice $\mathcal{L}$ if it is defined (specified) for all $\vec{r} \in \mathcal{L}$ and is either undefined or has value 0 for any point $\vec{r} \notin \mathcal{L}$. A signal is periodic with respect to a lattice $\mathcal{L}$ if $x(\vec{r})=x(\vec{r}+\vec{\xi})$ for all $\vec{\xi} \in \mathcal{L}$, and if $V$ is a generator of $\mathcal{L}$ then we say $x$ is periodic with respect to $V$.

An integer matrix $\mathrm{M}$ is called unimodular if $|\operatorname{det} M|=1$ and this occurs iff $M^{-1}$ is also an integer matrix. The following lemmas summarize basic results regarding lattices [3].

Lemma 1: $\mathcal{L}_{U}$ is a sublattice of $\mathcal{L}_{V}$, that is, $\mathcal{L}_{U} \subset \mathcal{L}_{V}$ iff $U=V M$ for some integer matrix $M$, and $\mathcal{L}_{U}=\mathcal{L}_{V}$ iff $U=V E$ for some unimodular matrix $E$.

Lemma 2: Points in a lattice form an abelian group under vector addition, and a sublattice is a subgroup. The translated sets of a sublattice, $\mathcal{L}_{U}+\vec{r}$ for $\vec{r} \in \mathcal{L}_{V}$, called cosets, form a group under set addition, called the quotient group and denoted $\mathcal{L}_{V} / \mathcal{L}_{U}$, and then $\left|\mathcal{L}_{V} / \mathcal{L}_{U}\right|=|\operatorname{det} M|$. As a consequence, the value of $|\operatorname{det} M|$ does not depend on the choice of generators for the lattices.

The density of a lattice, denoted by $\rho(\mathcal{L})$, is the number of lattice points per unit volume, and is given by $\rho(\mathcal{L})=\frac{1}{|\operatorname{det} V|}$. Thus, a sublattice is sparser (less dense) than the parent lattice.

Sampling patterns with variable spatial density arise by combining points from different sublatticesor their cosetsin different regions. In Figure 2, the central region contains points from a dense lattice, and the outer region contains only points in a sublattice. We refer to this as a multiresolution sampling pattern.

For a lattice $\mathcal{L}_{V}$, a corresponding unit cell $\mathcal{U}_{V} \subset \mathcal{R}^{D}$ is a set of points such that, for all $\vec{r} \in$ $\mathcal{R}^{D}$,there is a unique vector $\vec{r}_{0} \in \mathcal{L}_{V}$ such that $\vec{r}-\vec{r}_{0} \in \mathcal{U}_{V}$. Two examples of unit cells are the sets $V[0,1)^{D}$, called the fundamental parallelepiped (FPD) of $V$, and $V\left[-\frac{1}{2}, \frac{1}{2}\right)^{D}$. Regarding the cosets (distinct translations) of $\mathcal{L}_{V}$ in $\mathcal{R}^{D}$, a unit cell constitutes a minimal complete collection of coset representatives. Addition of points in the unit cell modulo the lattice corresponds to the quotient group $\mathcal{R}^{D} / \mathcal{L}_{V}$. The volume of a unit cell is $|\operatorname{det} V|$, the reciprocal of the density of the lattice.

If $\mathcal{L}_{U} \subset \mathcal{L}_{V}$, we denote by $\mathcal{J}(U, V)$ a complete set of coset representatives. That is, for every coset of $\mathcal{L}_{U}$ in $\mathcal{L}_{V}$, there is a unique element $\vec{r} \in \mathcal{J}(U, V)$ such that the coset can be expressed as $\mathcal{L}_{U}+\vec{r}$. We call such $\mathcal{J}(U, V)$ an index set.

Lemma 3: If $\mathcal{L}_{U} \subset \mathcal{L}_{V}$ with $U=V M$, then $\mathcal{J}(U, V)$ is a valid index set iff it has the form: $\mathcal{J}(U, V)=\mathcal{U}_{U} \cap \mathcal{L}_{V}$

for some unit cell $\mathcal{U}_{U}$ of $U$. Moreover, every index set contains exactly $|\operatorname{det} M|$ points. 


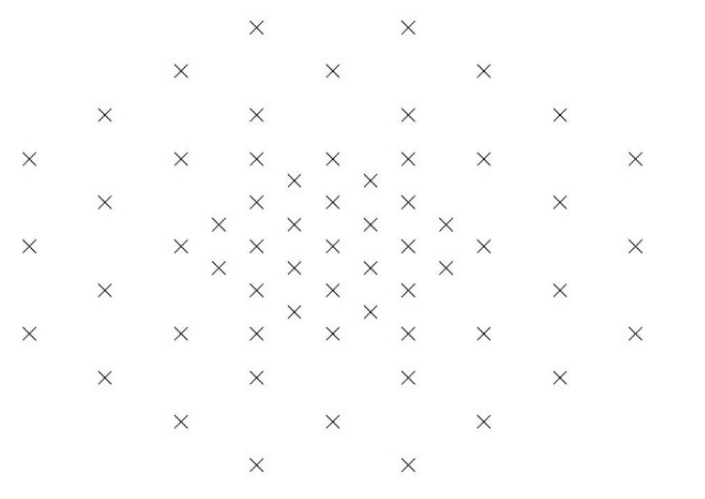

Figure 2: A multiresolution sampling pattern.

The quotient group $\mathcal{L}_{V} / \mathcal{L}_{U}$ can be interpreted as addition of points in an index set $\mathcal{J}(U, V)$ modulo the lattice $\mathcal{L}_{U}$. Figure 3 shows a sublattice (with points marked with 'o') and a coset (with points marked with ' $\times$ ') that together comprise a denser lattice. In this case, the quotient group contains two elements.

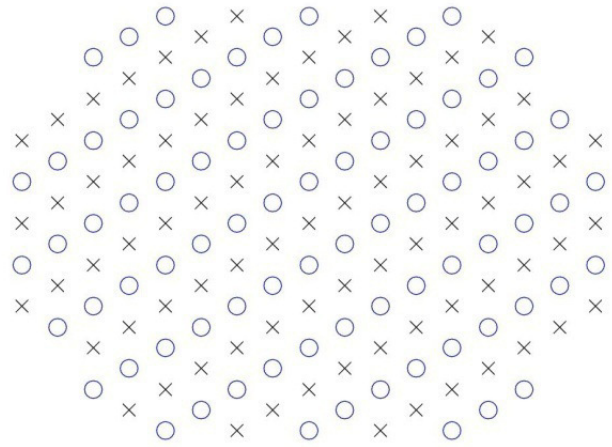

Figure 3: A sublattice ('o') and its coset (' $x$ ') together forming a denser lattice.

For a matrix $A$, let $\tilde{A}=A^{-T}$. Given a lattice $\mathcal{L}_{V}$, we call $\mathcal{L}_{\widetilde{V}}$ the reciprocal lattice, and $\mathcal{U}_{\widetilde{V}}$ the reciprocal unit cell. The density of the reciprocal lattice is the reciprocal of the density of the original lattice. A dense lattice will correspond to small unit cells, a sparse reciprocal lattice and large reciprocal unit cells. Note that if $U=V M$ then $\widetilde{V}=\widetilde{U} M^{T}$. Hence, whereas $\mathcal{L}_{U} \subset \mathcal{L}_{V}$, we get $\mathcal{L}_{\widetilde{V}} \subset \mathcal{L}_{\widetilde{U}}$. Also, $\left|\mathcal{L}_{V} / \mathcal{L}_{U}\right|=\left|\mathcal{L}_{\widetilde{U}} / \mathcal{L}_{\widetilde{V}}\right|=|\operatorname{det} M|$.

\subsection{Signals on Lattices with Finite Extent}

Consider a signal on a lattice $\mathcal{L}_{V}$ with a finite number of nonzero values, at points that are confined to within a unit cell $\mathcal{U}_{U}$ of a sublattice. In this case, the signal has support within an index set $\mathcal{J}(U, V)$, and by extending these values periodically with respect to $\mathcal{L}_{U}$, we can now interpret this as a signal over the quotient group $\mathcal{L}_{V} / \mathcal{L}_{U}$. There exists a finite Fourier transform that can be defined for such signals, and the focus of this paper is its study. We call this Fourier transform the multiresolution lattice discrete Fourier transform (MRL-DFT).

The MRL-DFT results in a frequency domain signal that has support in $\mathcal{L}_{\widetilde{U}}$ and is $\mathcal{L}_{\widetilde{V}}$-periodic, i.e., a signal over $\mathcal{L}_{\widetilde{U}} / \mathcal{L}_{\widetilde{V}}$. The spectrum is thus identified by its values over an index set $\mathcal{J}(\widetilde{\mathrm{V}}, \widetilde{\mathrm{U}})$, 
the complete set of points in the reciprocal lattice for $U$ that lie within a reciprocal unit cell for $V$. This corresponds to a generalized result of sampling theory: the density of samples in the original (here, spatial) domain determines the extent (bandwidth) in the frequency domain, and the extent in the original domain (spatial size) determines the density of points in the frequency domain.

Associated with this, we develop several multirate signal processing techniques, such as decimation and interpolation, polyphase components, filtering (i.e., convolution) and associated frequency domain concepts. The multiresolution approach is to consider not only a single sparse (low resolution) sublattice of a dense (high resolution) lattice, but also chains of intervening lattices with intermediate densities, that is:

$$
\mathcal{L}_{U} \subset \mathcal{L}_{U_{1}} \subset \mathcal{L}_{U_{2}} \subset \cdots \subset \mathcal{L}_{U_{k}} \subset \mathcal{L}_{V}
$$

This study facilitates development of applications such as representing signals with varying sampling densities in different regions of space, formulating fast algorithms for computing convolution and Fourier transforms, and obtaining techniques for regridding, i.e., interpolating data measured at possible irregularly sampled points to multiresolution lattices.

A theory of multidimensional multirateprocessing has been developed, for example in [3]. However, in that context, it is traditional to represent the signals as having support in $Z^{D}$. Consider a lattice $\mathcal{L}_{V} \subset \mathcal{R}^{D}$ with an identified generator $V$. As $\mathcal{L}_{V}=V Z^{D}$, if $x$ is a signal over $\mathcal{L}_{V}$ then the value $x(\vec{r})$ at a point $\vec{r} \in \mathcal{L}_{V}$ can be associated with the unique integer index vector $\vec{n}$ such that $\vec{r}=V \vec{n}$. Let us introduce the following notation:

$$
x_{V}[\vec{n}]=x(V \vec{n})
$$

We call this form lexicographic indexing. An integer vector $\vec{n}$ is associatedwith a point in physical space through the matrix $V$. The problem isphysical dimension, distance and direction are lost. Consider a discrete signal on a rectangulargrid, with a rectangular pixel (or voxel) having respective dimensions $\Delta x_{i}, 1 \leq i \leq D$. Then wecan take $V=\operatorname{diag}\left\{\Delta x_{i}\right\}$. But these dimensions are hidden in the lexicographic indexing form.As another example, consider changing the lattice generator, say $V^{\prime}=V E$ where $E$ is unimodular. Then:

$$
x_{V,}[\vec{n}]=x_{V}[E \vec{n}]
$$

Since $E Z^{D}=Z^{D}$, this change of generator matrix corresponds to a permutation, or rearrangement of lexicographic indices. But the underlying signal, and the features and information contained in the signal, has not actually changed. This artificial indexing can mask spatial correlations which often important to consider.

The act of decimation corresponds to keeping only points within a sublattice, say generated by $U=V M$. Decimation is typically expressed in lexicographic form as:

$$
x_{U}[\vec{n}]=x_{V}[M \vec{n}]
$$

This operation is called decimation with respect to the integer matrix $M$. This suggests a significant change in signal, and certainly the notion of spatial proximity is lost. In a physical sense, though, the only true change in the signal is that some values are discarded (effectively, set to 0 ), but the physical positions of the retained points do not change at all. The geometric structures of the underlying lattices are lost as well, because the change from say $\mathcal{L}_{V}$ to $\mathcal{L}_{U}$ as the underlying lattice of support is masked by forcing indexing via points in $Z^{D}$. Consider the case of rectangular sampling mentioned above. Decimating in the 1-direction by a factor of 2, basically changing $\Delta x_{1}$ to $2 \Delta x_{1}$ but no change in the other directions, is a nonuniform change of the shape 
of a pixel (voxel) that is not evident in lexicographic form. Procedures to design filtering structures, as in [14], and fast algorithms for spectral analysis, as in [15], are usually cast in lexicographic form, and thus are often not tied to the actual geometric structure of the underlying signals that need to be processed in a number of applications.

Here we develop a multirate signal processing approach retaining the physical indices, while relating results back to the traditional lexicographic index form. Using lexicographic notation can help determine techniques for data storage and retrieval of the signals of interest, but the exploration and development of signal processing techniques is facilitated by working with the physical coordinates.

\section{FOURIER TRANSFORMS}

There are generally four forms of the Fourier transform, corresponding to signals that are either continuous or discrete, and aperiodic (infinite in extent) or periodic (finite in support and extended periodically). If the starting point is the continuous aperiodic form, the others can be obtained by sampling in the spatial or frequency domains, or both. Here we obtain formulas for all, preserving the physical nature of the spatial or frequency domain coordinates, with connections to the normalized (lexicographic) forms.

\subsection{Multidimensional forms of CTFT, DTFT and Fourier Series}

We start with the continuous aperiodic form of the Fourier transform, where the spatial and frequency domains are both $\mathcal{R}^{D}$. This is a multidimensional form of the continuous-time Fourier transform (CTFT). For a signal $g: \mathcal{R}^{D} \rightarrow \mathcal{C}$ the Fourier transform is a signal $G: \mathcal{R}^{D} \rightarrow \mathcal{C}$ where:

$$
\begin{aligned}
G(\vec{f}) & =\int_{\mathcal{R}^{D} .} g(\vec{r}) e(-<\vec{f}, \vec{r}>) d \vec{r} \\
g(\vec{r}) & =\int_{\mathcal{R}^{D} .} G(\vec{f}) e(<\vec{f}, \vec{r}>) d \vec{f}
\end{aligned}
$$

Our approach to obtaining the other forms of the Fourier transform is to represent a discrete signal as a superposition of impulses, and pass it through the continuous form of the Fourier transform.

Note that $\delta\left(\vec{r}-\overrightarrow{r_{0}}\right) \rightarrow e\left(-<\vec{f}, \overrightarrow{r_{0}}>\right)$ and $e\left(<\overrightarrow{f_{0}}, \vec{r}>\right) \rightarrow \delta\left(\vec{f}-\overrightarrow{f_{0}}\right)$.

Take a signal over a lattice, $g: \mathcal{L}_{V} \rightarrow \mathcal{C}$, with generator matrix $V$.The corresponding frequency domain will be continuous, but periodic with respect to the reciprocal lattice, that is, the spectrum is a signal $G: \mathcal{R}^{D} / \mathcal{L}_{\widetilde{V}} \rightarrow \mathcal{C}$. To see this, start with the impulsive signal:

$$
g_{0}(\vec{r})=\sum_{\vec{\xi} \in \mathcal{L}_{V}} g(\vec{\xi}) \delta(\vec{r}-\vec{\xi})
$$

Substitution into the Fourier transform yields a spectrum that is a superposition of spectral functions of the form

$$
\phi_{\vec{\xi}}(\vec{f})=e(-<\vec{f}, \vec{\xi}>)
$$

where $\vec{\xi} \in \mathcal{L}_{V}$. Note $\vec{\xi}=V \vec{n}$ for some $\vec{n} \in Z^{D}$ and $\langle\vec{f}, V \vec{n}\rangle=\left\langle V^{T} \vec{f}, \vec{n}\right\rangle$. Therefore, $\phi_{\vec{\xi}}\left(\vec{f}+V^{-T} \vec{m}\right)=\phi_{\vec{\xi}}(\vec{f})$ for $\vec{m} \in Z^{D}$ and hence $\phi_{\vec{\xi}}$ is periodic with respect to the reciprocal lattice $\mathcal{L}_{\widetilde{V}}$, and the spectrum is completely determined by its values in one reciprocal unit 
cell $\mathcal{U}_{\widetilde{V}}$. We thus obtain the multidimensional form of the so-called discrete-time Fourier transform (DTFT) for signals over a lattice:

$$
\begin{gathered}
G(\vec{f})=\sum_{\vec{r} \in \mathcal{L}_{V}} g(\vec{r}) e(-<\vec{f}, \vec{r}>) \\
g(\vec{r})=|\operatorname{det} V| \int_{\mathcal{U}_{\tilde{V}}} G(\vec{f}) e(<\vec{f}, \vec{r}>) d \vec{f}
\end{gathered}
$$

Substituting $\vec{\xi}=V \vec{n}, \vec{n} \in Z^{D}$, and $\vec{f}=\tilde{V} \vec{u}, \vec{u} \in\left[-\frac{1}{2}, \frac{1}{2}\right)^{D}$, yields the more standard, normalized (lexicographic) form of the multidimensional DTFT:

$$
\begin{gathered}
G[\vec{u}]=\sum_{\vec{n} \in \mathcal{Z}^{D}} g[\vec{n}] e(-<\vec{u}, \vec{n}>) \\
g[\vec{n}]=\int_{\vec{u} \in\left[-\frac{1}{2}, \frac{1}{2}\right)^{D}} G[\vec{u}] e(<\vec{u}, \vec{n}>) d \vec{u}
\end{gathered}
$$

A similar approach can be used to obtain the multidimensional form of the Fourier series. We start with a signal whose spectrum has support on the reciprocal lattice of $V$ in the frequency domain. By representing the spectrum as a sum of impulses located at the reciprocal lattice points, we find the signal in the spatial domain is continuous but periodic with respect to the original lattice. Thus, continuous lattice-periodic signals $g: \mathcal{R}^{D} / \mathcal{L}_{V} \rightarrow \mathcal{C}$ have discrete spectra with support on the reciprocal lattice, $G: \mathcal{L}_{\widetilde{V}} \rightarrow \mathcal{C}$, with Fourier transform given by:

$$
\begin{gathered}
G(\vec{f})=\frac{1}{|\operatorname{det} V|} \int_{\mathcal{U}_{V}} g(\vec{r}) e(-<\vec{f}, \vec{r}>) d \vec{r} \\
g(\vec{r})=\sum_{\vec{f} \in \mathcal{L}_{\widetilde{V}}} G(\vec{f}) e(<\vec{f}, \vec{r}>)
\end{gathered}
$$

Expressing both the spatial and frequency domains in normalized (lexicographic) form results in the more conventional multidimensional Fourier series formulas:

$$
\begin{gathered}
G[\vec{k}]=\int_{\vec{\zeta} \in\left[-\frac{1}{2}, \frac{1}{2}\right)^{D}} g[\vec{\zeta}] e(-<\vec{k}, \vec{\zeta}>) d \vec{\zeta} \\
g[\vec{\zeta}]=\sum_{\vec{k} \in Z^{D}} G[\vec{k}] e(<\vec{k}, \vec{\zeta}>)
\end{gathered}
$$

In normalized form, the spatial signal is viewed in $\mathcal{R}^{D} / Z^{D}$ and the spectrum is a signal over $Z^{D}$.

\subsection{MRL-DFT}

We now present the multiresolution-lattice discrete Fourier transform (MRL-DFT), which is a multidimensional form of the DFT that retains the geometric structure of space and frequency.

The MRL-DFT applies to signals with support on one lattice, periodic with respect to a sublattice. To justify the formulation, we start with a special case, which is signals over $Z^{D}$ that are periodic 
with respect to an integer matrix, $N$. This yields the conventional form of the multidimensional DFT, which is in lexicographic form.

In one dimension, the DFT applies to a time-domain signal where the time is integer valued and periodic with period N. To apply Fourier analysis, the time domain must be a group, and in fact the group is the cyclic group $Z_{N}=\{0,1, \cdots, N-1\}$ (i.e., modulo- $N$ addition), which is isomorphic to the quotient group $Z / N Z$, which we recognize as a simple lattice quotient group. The frequency domain is also $Z_{N}$. The parameter $N$ is called the radix of the DFT. The simplest multidimensional form assumes a signal over an $N_{1} \times N_{2} \times \cdots \times N_{D}$ grid, and the DFT can be applied separately in each dimension, resulting in the formulas:

$$
\begin{gathered}
G[\vec{k}]=\sum_{0 \leq n_{i} \leq N_{i}-1} g[\vec{n}] e\left(-\sum \frac{n_{i} k_{i}}{N_{i}}\right) \\
g[\vec{n}]=\frac{1}{N_{1} N_{2} \cdots N_{D}} \sum_{0 \leq k_{i} \leq N_{i}-1} G[\vec{k}] e\left(\sum \frac{n_{i} k_{i}}{N_{i}}\right)
\end{gathered}
$$

Define the matrix $N=\operatorname{diag}\left\{N_{1}, N_{2}, \cdots, N_{D}\right\}$. The scaling factor $N_{1} N_{2} \cdots N_{D}=|\operatorname{det} N|$, and also:

$$
<\vec{k}, N^{-1} \vec{n}>=k^{T} N^{-1} n=\sum \frac{n_{i} k_{i}}{N_{i}}
$$

Let us define $\mathcal{J}_{N}=\left\{\vec{n}: 0 \leq n_{i} \leq N_{i}-1\right\}$. This set is a minimal set of coset representatives of $\mathcal{L}_{N}$ in $Z^{D}$. The sums are both taken over this set. In generalizing this to non-diagonal integer matrices, care must be taken because, in this case, $N=N^{T}$. In order to arrive at the correct form for a general integer matrix, we need a mathematical result.In what follows, for an integer matrix $M$, let $J_{M}$ be an index set for $M$ in the integer lattice, that is, $J_{M}=\mathcal{U}_{M} \cap Z^{D}$ for any unit cell $\mathcal{U}_{M}$. For $\vec{n}, \vec{k} \in Z^{D}$, and $D \times D$ invertible integer matrix $N$, define:

$$
\phi_{N}(\vec{n}, \vec{k})=e\left(<\vec{k}, N^{-1} \vec{n}>\right)=e(<\widetilde{N} \vec{k}, \vec{n}>)
$$

Lemma 4: For $\vec{k} \in Z^{D}, \phi_{N}(\cdot, \vec{k})$ is $\mathcal{L}_{N}$-periodic, and for $\vec{n} \in Z^{D}, \phi_{N}(\vec{n}, \cdot)$ is $\mathcal{L}_{N^{T}}$-periodic. Moreover, the following orthogonality conditions hold[1]:

$$
\begin{aligned}
& \frac{1}{|\operatorname{det} N|} \sum_{\vec{n} \in J_{N}} \phi_{N}(\vec{n}, \vec{k})=\left\{\begin{array}{l}
1, \vec{k} \in \mathcal{L}_{N^{T}} \\
0, \vec{k} \notin \mathcal{L}_{N^{T}}
\end{array}\right. \\
& \frac{1}{|\operatorname{det} N|} \sum_{\vec{k} \in J_{N} T} \phi_{N}(\vec{n}, \vec{k})=\left\{\begin{array}{l}
1, \vec{n} \in \mathcal{L}_{N} \\
0, \vec{n} \notin \mathcal{L}_{N}
\end{array}\right.
\end{aligned}
$$

Using this Lemma, we can obtain the DFT formulation simply as a standard (finite-dimensional) orthogonal expansion:

Definition 2: The discrete Fourier transform (DFT) with respect to an integer matrix $N$ maps spatial domain signals over $Z^{D} / N Z^{D}$ to frequency domain signals over $Z^{D} / N^{T} Z^{D}$ as follows: 


$$
\begin{gathered}
G[\vec{k}]=\sum_{\vec{n} \in J_{N}} g[\vec{n}] e\left(-<\vec{k}, N^{-1} \vec{n}>\right) \\
g[\vec{n}]=\frac{1}{|\operatorname{det} N|} \sum_{\vec{k} \in \mathcal{J}_{N^{T}}} G[\vec{k}] e\left(<\vec{k}, N^{-1} \vec{n}>\right)
\end{gathered}
$$

Even if we started with a signal truly on an integer lattice, this formulation gives a normalized (lexicographic) frequency domain. We would expect the spectrum to lie at points on a lattice generated by $\widetilde{N}$. But for a general integer matrix $N, \widetilde{N}$ is not an integer matrix, and instead this form of the DFT indexes the frequency domain at integer vectors in the set $\mathcal{J}_{N}$.

We propose the MRL-DFT as a formulation that preserves the geometric features of the spatial and frequency domains. The proper form of the equations is provided by the following theorem.

Theorem 5: Let $\mathcal{L}_{U} \subset \mathcal{L}_{V}$ be lattices in $\mathcal{R}^{D}$. Then the Fourier transform of a spatial signal over $\mathcal{L}_{V} / \mathcal{L}_{U}$ is a frequency domain signal over $\mathcal{L}_{\widetilde{U}} / \mathcal{L}_{\widetilde{V}}$, and the transform and inverse transform formulas are given by:

$$
\begin{aligned}
& G(\vec{f})=\sum_{\vec{r} \in \mathcal{J}\left(\mathcal{L}_{U}, \mathcal{L}_{V}\right)} g(\vec{r}) e(-<\vec{f}, \vec{r}>) \\
& g(\vec{r})=\frac{1}{|\mathcal{J}|} \sum_{\vec{f} \in \mathcal{J}\left(\mathcal{L}_{\tilde{V}}, \mathcal{L}_{\tilde{U}}\right)} G(\vec{f}) e(<\vec{f}, \vec{r}>)
\end{aligned}
$$

We call this the multiresolution lattice discrete Fourier transform (MRL-DFT).

Proof: Since $g(\vec{r})$ is $\mathcal{L}_{U}$-periodic, the previous discussion yields that its Fourier transform $G(\vec{f})$ has support in $\mathcal{L}_{\widetilde{U}}$. Then the Fourier series formulation yields:

$$
G(\vec{\zeta})=\frac{1}{|\operatorname{det} U|} \int_{\mathcal{U}_{U}} g_{0}(\vec{r}) e(-<\vec{\zeta}, \vec{r}>) d \vec{r}
$$

where $g_{0}$ is a signal over continuous-space, but is actually concentrated only at points in the lattice $\mathcal{L}_{V}$. In fact, $g_{0}$ is an impulsive function, with impulses located at the lattice points. The integral becomes a sum at points $\mathcal{U}_{U} \cap \mathcal{L}_{V}$, which is $\mathcal{J}\left(\mathcal{L}_{U}, \mathcal{L}_{V}\right)$, and we obtain the MRL-DFT in the theorem, up to the scaling factor $|\operatorname{det} U|$. Now examine the reverse situation: start with $g(\vec{r})$ having support on the lattice $\mathcal{L}_{V}$ with a spectrum $G(\vec{f})$ that is $\mathcal{L}_{\widetilde{V}}$-periodic. The generalized inverse DTFT yields an integral over the continuous frequency domain region $\mathcal{U}_{\widetilde{V}}$, but the integrand is impulsive since it lies at discrete lattice points only, and the integral becomes the inverse MRL-DFT formula, except that the scaling factor $1 /|\mathcal{J}|$ is replaced with $|\operatorname{det} V|$. To account for the scaling factors, we recognize that in Fourier transform and inverse transform formulas, what is significant is not the individual scaling factors, but their product. We can rescale the defined spectrum, for example, and since $|\operatorname{det} 3|=\frac{|\operatorname{det} U|}{|\operatorname{det} V|}$ the proposed MRL-DFT and inverse MRL-DFT formulas are confirmed.

We note that if both the spatial and frequency domains in the MRL-DFT are expressed in lexicographic form, with $U=V N$ for an integer matrix $N$, the MRL-DFT reduces to the standard DFT described previously. 


\subsection{Properties of the MRL-DFT}

As with any Fourier transform, we expect certain properties to hold, such as convolution in one domain corresponds to multiplication in the other domain. All properties listed here are special cases of properties of abstract Fourier transforms, and can be derived in that context, for example through group theory. Therefore we do not provide complete proofs for all properties listed here, but highlight some examples to illustrate the application of lattice theory. Here, $\mathcal{F}\{x\}$ denotes the MRL-DFT of $x$, and $\mathcal{F}^{-1}\{X\}$ denotes the inverse MRL-DFT of $X$.

Definition 3: Let $h$,gbe signals over $\mathcal{L}_{V} / \mathcal{L}_{U}$. Then the convolution $y=h * g$ is a signal over $\mathcal{L}_{V} / \mathcal{L}_{U}$ and is given by:

$$
y(\vec{r})=\sum_{\vec{s} \in \mathcal{J}\left(\mathcal{L}_{U}, \mathcal{L}_{V}\right)} h(\vec{s}) g(\vec{r}-\vec{s})
$$

and the correlation $\phi_{h g}$ is a signal over $\mathcal{L}_{V} / \mathcal{L}_{U}$ given by:

$$
\phi_{h g}(\vec{r})=\sum_{\vec{s} \in \mathcal{J}\left(\mathcal{L}_{U}, \mathcal{L}_{V}\right)} h(\vec{r}+\vec{s}) g^{*}(\vec{s})
$$

In each case, the terms in the sum are $\mathcal{L}_{U}$-periodic. Convolution and correlation in the frequency domain are defined similarly, i.e., without any scaling factors in the sums.

Lemma 6: If $x(\vec{r})=g^{*}(-\vec{r})$ then $\phi_{h g}=h * x$.

Lemma 7: $\mathcal{F}\left\{x^{*}(\vec{r})\right\}=X^{*}(-\vec{f})$ and $\mathcal{F}\left\{x^{*}(-\vec{r})\right\}=X^{*}(\vec{f})$.

Theorem 8: Convolution in the spatial domain corresponds to multiplication in the frequency domain, and conversely, specifically:

$$
\begin{gathered}
\mathcal{F}\{h * g\}=H \cdot G \\
\mathcal{F}\{h \cdot g\}=|\mathcal{J}| H * G
\end{gathered}
$$

Corollary 9: The MRL-DFT of the correlation function $\phi_{h g}$ is $H \cdot G^{*}$.

Now matching $\phi_{h g}(\overrightarrow{0})$ to the inverse MRL-DFT of $H \cdot G^{*}$ evaluated at $\overrightarrow{0}$ results in the following:

Corollary 10: Parseval's theorem for the MRL-DFT:

$$
\sum_{\vec{r} \in \mathcal{J}\left(\mathcal{L}_{U}, \mathcal{L}_{V}\right)} h(\vec{r}) g^{*}(\vec{r})=\frac{1}{|\mathcal{J}|} \sum_{\vec{f} \in \mathcal{J}\left(\mathcal{L}_{\widetilde{V}}, \mathcal{L}_{\widetilde{U}}\right)} H(\vec{f}) G^{*}(\vec{f})
$$

We omit the algebraic details of these results, except to point out two fundamental concepts that underlie the proof of the convolution theorem. The first is the factorization of the exponential function:

$$
e(<\vec{f}, \vec{r}>)=e(<\vec{f}, \vec{r}-\vec{s}>) \cdot e(<\vec{f}, \vec{s}>)
$$

The second deals with summing over the index set. The difficulty is that if $\vec{r}, \vec{s} \in \mathcal{J}\left(\mathcal{L}_{U}, \mathcal{L}_{V}\right)$, in general, $\vec{r}-\vec{s}$ is not in the index set. However, the signals and the exponential functions appearing 
in the sums are $\mathcal{L}_{U}$-periodic. If $\vec{\xi} \in \mathcal{L}_{V}$, let $(\vec{\xi})_{U} \in \mathcal{J}\left(\mathcal{L}_{U}, \mathcal{L}_{V}\right)$ be the unique point in the index set such that $\vec{\xi}-(\vec{\xi})_{U} \in \mathcal{L}_{U}$. We can think of $(\vec{\xi})_{U}$ as the value of $\vec{\xi}$ modulo the sublattice $\mathcal{L}_{U}$. Then for each $\vec{s}$, the mapping $\vec{r} \rightarrow(\vec{r}-\vec{s})_{U}$ is a one-to-one mapping of $\mathcal{J}\left(\mathcal{L}_{U}, \mathcal{L}_{V}\right)$ onto itself.

\section{Multiresolution Lattices}

This section lays the groundwork for signal processing on multiresolution lattices. Specifically, we consider chains of lattices of varying densities, say $\mathcal{L}_{U} \subset \mathcal{L}_{W} \subset \mathcal{L}_{V}$. Let $W=V N, U=W Q$, so that $U=V M$ with $M=N Q$. To avoid the trivial case, where $\mathcal{L}_{W}$ equals either $\mathcal{L}_{U}$ or $\mathcal{L}_{V}$, we require that neither $N$ nor $Q$ be unimodular. Therefore, we can generate intervening sublattices if we can obtain non-trivial factorization of integer matrices. Such factorizations, in fact, will allow us to describe the structure of $\mathcal{L}_{V} / \mathcal{L}_{U}$.

Another issue we must address is that, in order to actually perform computations such as convolution, correlation or MRL-DFT, we need to be able to obtain valid index sets for specific lattices. It is not obvious how to find a specific set of points that is a valid choice for $\mathcal{J}\left(\mathcal{L}_{U}, \mathcal{L}_{V}\right)$. The next section describes an important mathematical result we will rely on.

\subsection{Smith form for integer matrices}

Proposition 11: Any $D \times D$ nonsingularinteger matrix $M$ can be factored as $M=E K F$ with $E, F$ unimodular, and $K=\operatorname{diag}\left\{k_{1}, k_{2}, \cdots, k_{D}\right\}$ witheach $k_{i}$ a positive integer. This form is not unique, but the $k_{i}$ 's are unique up to a permutation. This is called the Smith form of an integer matrix. [16]

Note that $|\operatorname{det} M|=\prod k_{i}$. This allows us to identify all possible combinations of $k_{i}$ 's. For example, if $M$ is $3 \times 3$ and $|\operatorname{det} M|=12$, the Smith form of $M$ will result in one of the following (up to a permutation): $(12,1,1),(6,2,1),(4,3,1)$, or $(3,2,2)$.

We now seek a factorization $M=N Q$ where neither $N$ nor $Q$ is unimodular. This hinges on the factorization of $K$. Let $K=A B$ where $A, B$ are diagonal matrices with positive integers on the diagonals. At the $i^{\text {th }}$ position, we must have $k_{i}=a_{i} b_{i}$. To avoid a trivial factorization, we require at least one $a_{i}>1$ and at least one $b_{i}>1$. For example if $K=\operatorname{diag}\{4,3,1\}$ we could take $A=\operatorname{diag}\{4,1,1\}, B=\operatorname{diag}\{1,3,1\} \quad$ or $\quad A=\operatorname{diag}\{2,3,1\}, B=\operatorname{diag}\{2,1,1\}, \quad$ or $A=\operatorname{diag}\{2,1,1\}, B=\operatorname{diag}\{2,3,1\}$, or $A=\operatorname{diag}\{1,3,1\}, B=\operatorname{diag}\{4,1,1\}$. There are no other possibilities! Then with $N=E A$ and $Q=B F$, we have a nontrivial factorization $M=N Q$.

Given the Smith form $M=E K F$, and $U=V M$, we can write $U^{\prime}=V^{\prime} K$ where $U^{\prime}=U F^{-1}, V^{\prime}=V E$. Since $F$ is unimodular, so is $F^{-1}$. Therefore, $U^{\prime}, V^{\prime}$ generate the same lattices as $U, V$, and we have found a representation of the lattices such that the generators are related by a diagonal integer matrix. But finding an index set then is straightforward. For example, if $K=\operatorname{diag}\{4,3,1\}$, then the entries in the index set are vectors of the form $V^{\prime} \vec{n}=V E \vec{n}$ where $\vec{n}=\left(n_{1}, n_{2}, 0\right)$ with $0 \leq$ $n_{1} \leq 3,0 \leq n_{2} \leq 2$. This yields the precise mathematical structure of the quotient group.

Theorem 12: If $U=V M$ and the Smith form of $M$ is $E K F$ with $K=\operatorname{diag}\left\{k_{1}, k_{2}, \cdots, k_{D}\right\}$, then the quotient group $\mathcal{L}_{V} / \mathcal{L}_{U}$ is isomorphic to the direct product of cyclic groups as follows:

$$
z_{k_{1}} \times z_{k_{2}} \times \cdots \times z_{k_{D}}
$$

It is well known that every finite abelian group is the direct product of cyclic groups. The significance of this theorem is that we can use the Smith form to find the particular decomposition for a lattice quotient group. The Smith form allows us to generate proper index 
sets, and to obtain integer matrix factorizations to identify and generate all intervening lattices between $\mathcal{L}_{U}$ and $\mathcal{L}_{V}$.

\subsection{The MRL-DFT viewed on lattice chains}

In this section, we consider $U=V M$, and lattices that lie between those generated by $U$ and $V$, i.e., we study lattice chains such as $\mathcal{L}_{U} \subset \mathcal{L}_{W} \subset \mathcal{L}_{V}$. Let $\mathcal{S}(U, V)$ denote the space of complex valued signals with support on $\mathcal{L}_{V}$ that are periodic with respect to $\mathcal{L}_{U}$. The MRL-DFT for such signals will be in $\mathcal{S}(\widetilde{V}, \widetilde{U})$. We will also use the notation $\mathcal{S}(V)$ to denote signals with support on $\mathcal{L}_{V}$ that are not periodic. If we start with a signal with finite number of nonzero values, but the points of support lie completely within some unit cell $\mathcal{U}_{U}$ of $U$, then we will consider it extended periodically with respect to $\mathcal{L}_{U}$ to apply the techniques developed here. We apply the same principle similarly in the frequency domain.

The first result is a common property of Fourier transforms: translation in either the spatial or frequency domain corresponds to a linear phase shift in the other domain.

Lemma 13: Let $x \in \mathcal{S}(U, V), \vec{r}_{0} \in \mathcal{L}_{V}, \vec{f}_{0} \in \mathcal{L}_{\widetilde{U}}$. Also let $X$ be the $\mathcal{L}_{V} / \mathcal{L}_{U}$ MRL-DFT of $x$. Then the MRL-DFT of $x\left(\vec{r}+\vec{r}_{0}\right)$ is $e\left(<\vec{f}, \vec{r}_{0}>\right) X(\vec{f})$ and the MRL-DFT of $e\left(-<\vec{f}_{0}, \vec{r}>\right) x(\vec{r})$ is $X\left(\vec{f}+\vec{f}_{0}\right)$.

Proof: The results follow from a straightforward application of factorizations of the exponential function such as:

$$
e\left(<\vec{f}, \vec{r}+\vec{r}_{0}>\right)=e(<\vec{f}, \vec{r}>) \cdot e\left(<\vec{f}, \vec{r}_{0}>\right)
$$

Take $\mathcal{L}_{U} \subset \mathcal{L}_{W} \subset \mathcal{L}_{V}$. Consider a signal $x \in \mathcal{S}(U, W) \subset \mathcal{S}(U, V)$, specifically $x$ is 0 for points in $\mathcal{L}_{V}$ that are not in $\mathcal{L}_{W}$. We can compute the MRL-DFT with respect to either $\mathcal{L}_{W}$ or $\mathcal{L}_{V}$ as the lattice of support. However, since the signal itself is essentially the same, we hope the resulting spectra will match. That is in fact what happens. This confirms our formulation truly preserves the physical nature of the signals under study. To prove it, we need the following.

Lemma 14: If $\mathcal{L}_{U} \subset \mathcal{L}_{W} \subset \mathcal{L}_{V}$, and we have valid index sets $\mathcal{J}(U, W), \mathcal{J}(\mathrm{W}, V)$, then $\mathcal{J}(U, V)=$ $\mathcal{J}(U, W)+\mathcal{J}(\mathrm{W}, V)$ is a valid index set. Moreover, if $\overrightarrow{0} \in \mathcal{J}(W, V)$ then the result of this construction is that $\mathcal{J}(U, W) \subset \mathcal{J}(U, V)$.

Theorem 15: Let $\mathcal{L}_{U} \subset \mathcal{L}_{W} \subset \mathcal{L}_{V}$, and $x \in \mathcal{S}(U, W) \subset \mathcal{S}(U, V)$. Let $X^{(W)}$ denote its $\mathcal{L}_{W} / \mathcal{L}_{U}$ MRL-DFT, and $X^{(V)}$ its $\mathcal{L}_{V} / \mathcal{L}_{U}$ MRL-DFT. Both have support on $\mathcal{L}_{\widetilde{U}}$, and for all $\vec{f} \in \mathcal{L}_{\widetilde{U}}$ :

$$
X^{(V)}(\vec{f})=X^{(W)}(\vec{f})
$$

This relation has the same form in lexicographic notation, that is

$$
X^{(V)}[\vec{k}]=X^{(W)}[\vec{k}] \quad \forall \vec{k} \in Z^{D}
$$

Proof: $X^{(V)}(\vec{f})$ is computed as a sum over $\mathcal{J}(U, V)$ for some index set. By Lemma 14, we can chose $\mathcal{J}(U, W), \mathcal{J}(\mathrm{W}, V)$, with $\overrightarrow{0} \in \mathcal{J}(W, V)$, so that $\mathcal{J}(U, V)=\mathcal{J}(U, W)+\mathcal{J}(\mathrm{W}, V)$. With this construction, the only point in $\mathcal{J}(W, V)$ that is also in $\mathcal{L}_{W}$ is $\overrightarrow{0}$, and every point in $\mathcal{J}(U, W)$ is in $\mathcal{L}_{W}$. Therefore, $\mathcal{J}(U, V) \cap \mathcal{L}_{W}=\mathcal{J}(U, W)$. Therefore, the signal $x$ is zero at all other points in $\mathcal{J}(U, V)$, so the sum over $\mathcal{J}(U, V)$ reduces to a sum over $\mathcal{J}(U, W)$ and the MRL-DFTs match. 
Since both spectra have support on the same lattice, their lexicographic forms are both related through the same matrix, $\widetilde{U}$, so the lexicographic forms match as well.

The next result is that restriction to a sublattice in space or frequency corresponds to periodicity in the other domain.

Theorem 16: Let $\mathcal{L}_{U} \subset \mathcal{L}_{W} \subset \mathcal{L}_{V}$ and $x \in \mathcal{S}(U, V)$. Then $x$ has support on the sublattice $\mathcal{L}_{W}$ iff its $\mathcal{L}_{V} / \mathcal{L}_{U}$ MRL-DFT is $\mathcal{L}_{\widetilde{W}}$-periodic, and $x$ is $\mathcal{L}_{W}$-periodic iff its $\mathcal{L}_{V} / \mathcal{L}_{U}$ MRL-DFT has support on $\mathcal{L}_{\widetilde{W}}$.

Proof: We prove the first statement. The second follows from the symmetry between the MRLDFT and its inverse. First assume $x$ is zero outside $\mathcal{L}_{W}$. Then by Theorem $15, X^{(V)}(\vec{f})=$ $X^{(W)}(\vec{f})$, but $X^{(W)}$ must be $\mathcal{L}_{\widetilde{W}}$-periodic. So this part of the theorem is trivial. Now assume, conversely, that $X^{(V)}(\vec{f})$ is $\mathcal{L}_{\widetilde{W}}$-periodic. That means $X^{(V)} \in \mathcal{S}(\widetilde{W}, \widetilde{U})$. Perform an inverse $\mathcal{L}_{W} / \mathcal{L}_{U}$ MRL-DFT to obtain a spatial domain signal $\tilde{x} \in \mathcal{S}(U, W)$. Initially, $\tilde{x}$ is only defined on points in the lattice $\mathcal{L}_{W}$. Now let us extend its domain of definition to $\mathcal{L}_{V}$ with the rule that $\tilde{x}(\vec{r})=0$ for $\vec{r} \notin \mathcal{L}_{W}$. Then $\tilde{X}^{(V)}=\tilde{X}^{(W)}$, and $X^{(V)}=\tilde{X}^{(W)}$. Therefore, $X^{(V)}=\tilde{X}^{(V)}$. Since the MRL-DFT is invertible, $x=\tilde{x}$.

We can now generalize the prior theorem to describe signals who support is restricted to a coset of a sublattice in the spatial domain, or a coset of a reciprocal sublattice in the frequency domain.

Corollary 17: Let $\mathcal{L}_{U} \subset \mathcal{L}_{W} \subset \mathcal{L}_{V}$, and $x \in \mathcal{S}(U, V)$. Then $x$ has support only on a coset $\mathcal{L}_{W}+\vec{r}_{0}$ for some $\vec{r}_{0} \in \mathcal{L}_{V}$ iff its MRL-DFT satisfies:

$$
X(\vec{f}+\vec{\zeta})=e\left(-<\vec{\zeta}, \vec{r}_{0}>\right) X(\vec{f})
$$

Similarly, the MRL-DFT has support only on a $\operatorname{coset} \mathcal{L}_{\widetilde{W}}+\vec{f}_{0}$ for some $\vec{f}_{0} \in \mathcal{L}_{\widetilde{U}}$ iff it satisfies:

$$
x(\vec{r}+\vec{\xi})=e\left(<\vec{f}_{0}, \vec{\xi}>\right) x(\vec{r})
$$

Proof: For the first part, the frequency domain property can be shown to be equivalent to the condition that $\left.Y(\vec{f})=e\left(<\vec{f}, \vec{r}_{0}\right\rangle\right) X(\vec{f})$ is $\mathcal{L}_{\widetilde{W}}$-periodic, and the previous theorem can be applied. Similarly for the second statement.

\subsection{Regridding: upsampling, downsampling and general lattice conversion}

Here we discuss changing the sampling structure in the spatial or frequency domain. In classical sampling theory, decimation in time causes aliasing in frequency, and upsampling in time causes imaging distortion in frequency. These results can be mitigated by filtering, which can be implemented via convolution in time or windowing in frequency. Similar results occur if the situations are reversed, which does arise in applications that involve spectral analysis. For example, in MRI, spectral measurements are regridded to convenient point sets via interpolation.

Results are given in the physical domains, in terms of MRL-DFT, and then are reduced to the lexicographic forms. We start with a lattice chain $\mathcal{L}_{U} \subset \mathcal{L}_{W} \subset \mathcal{L}_{V}$ and assume a fixed choice of index sets, each of which are assumed to contain $\overrightarrow{0}$ where:

$$
\mathcal{J}(U, V)=\mathcal{J}(U, W)+\mathcal{J}(\mathrm{W}, V)
$$

By default, we use generator matrices $U, W, V$ where $U=W N, W=V Q$, and hence $U=V M$ with $M=N Q$. Also, we start with a signal $x \in \mathcal{S}(U, V)$ (periodic case) or $x \in \mathcal{S}(V)$ (aperiodic case). 
Definition 4: If $x$ has support on $\mathcal{L}_{V}$, decimation (downsampling) of $x$ to $\mathcal{L}_{W}$, denoted $y=$ $\left(\mathcal{L}_{V} \searrow \mathcal{L}_{W}\right) x$, is the restriction of $x$ to the lattice $\mathcal{L}_{W}$. That is $y=x$ at points in $\mathcal{L}_{W}$, but we set $y=0$ at points in $\mathcal{L}_{V}$ not in $\mathcal{L}_{W}$.

Definition 5: If $x$ has support on $\mathcal{L}_{W}$, upsampling of $x$ to $\mathcal{L}_{V}$, denoted $y=\left(\mathcal{L}_{W} \nearrow \mathcal{L}_{V}\right) x$, expands the domain of $x$ to $\mathcal{L}_{V}$ by specifying $y=x$ at points in $\mathcal{L}_{W}$ and $y=0$ at points in $\mathcal{L}_{V}$ not in $\mathcal{L}_{W}$.

Note that decimation does significantly change a signal, in that it discards a set of values. Upsampling does not truly change a signal. It is more a technical change that allows us to consider a denser lattice of support, but the additional values are all 0 . Based on the previous discussion, this does not actually change the MRL-DFT, and yet we expect an imaging phenomenon. In fact, a signal in $\mathcal{S}(U, V)$ normally has a spectrum that is completely specified by its support in a reciprocal unit cell $\mathcal{U}_{\widetilde{V}}$. However, because its MRL-DFT matches that for a signal in $\mathcal{S}(U, W)$, this spectrum appears in the smaller region $\mathcal{U}_{\widetilde{W}}$, periodically extended via the dense reciprocal lattice $\mathcal{L}_{\widetilde{U}}$. With $W=V Q$, it turns out there are $|\operatorname{det} Q|$ copies of the spectrum that appear in $\mathcal{U}_{\widetilde{V}}$, and these copies are seen because changing to a denser lattice in the spatial domain corresponds to a wider range of support in the frequency domain.

We expect the decimation process to introduce aliasing, which actually changes the spectral values. To obtain this result, note that decimation can be achieved by multiplying the original signal by a 1-0 function. Specifically, for a set $\mathcal{A}$, let $\chi_{\mathcal{A}}$ be a signal that is 1 in $\mathcal{A}$ and 0 outside of $\mathcal{A}$; this is called the indicator function. Then direct computation of the $\mathcal{L}_{V} / \mathcal{L}_{U}$ MRL-DFT yields that the spectrum of $\chi_{\mathcal{L}_{W}}$ is $|\operatorname{det} N| \chi_{\mathcal{L}_{\widetilde{W}}}$ where $U=W N$. Decimation corresponds to multiplying in the spatial domain by the indicator function of the sparser lattice, and thus the frequency domain equivalent is governed by a convolution with an indicator function. This results in the following formula, which is the aliasing phenomenon:

Theorem 18: If $y=\left(\mathcal{L}_{V} \searrow \mathcal{L}_{W}\right) x$, then the MRL-DFT of $y$ is related to the MRL-DFT of $x$ via:

$$
Y(\vec{f})=\frac{1}{|\mathcal{J}(\mathrm{W}, V)|} \sum_{\vec{\zeta} \in \mathcal{J}(\widetilde{V}, \widetilde{W})} X(\vec{f}+\vec{\zeta})
$$

The lexicographic definitions of decimation and upsampling, and the corresponding frequency domain formulas, are obtained by recognizing the matrices that relate the various lattices. For example, $U=V M$ and $\tilde{V}=\widetilde{U} M^{T}$. With $W=V Q$ and $y=\left(\mathcal{L}_{V} \searrow \mathcal{L}_{W}\right) x$ :

$$
y_{W}[\vec{n}]=y(W \vec{n})=y(V Q \vec{n})=x(V Q \vec{n})=x_{V}[Q \vec{n}]
$$

Here we have been careful to define the physical matrix associated with the lexicographic notation. The more traditional form is to omit this, and we obtain the standard formula[3] for decimation of signals on $Z^{D}$ with respect to an integer matrix $Q$, denoted as $y=(\downarrow Q) x$, via

$$
y[\vec{n}]=x[Q \vec{n}]
$$

Upsampling to a denser lattice, as defined here, becomes, in lexicographic form, equivalent to upsamplinga signal on $Z^{D}$ with respect to an integer matrix $Q$, denoted $y=(\uparrow Q) x$ :

$$
y[\vec{n}]= \begin{cases}x\left[Q^{-1} \vec{n}\right] & \text { if } \vec{n} \in Q Z^{D} \\ 0 & \text { if } \vec{n} \notin Q Z^{D}\end{cases}
$$


The following gives the lexicographic forms for aliasing and imaging in the frequency domain. Note that $\widetilde{W}=\widetilde{U} N^{T}, \widetilde{V}=\widetilde{W} Q^{T}$.

Corollary 19: Let $X[\vec{k}]=X(\widetilde{U} \vec{k}), Y[\vec{k}]=Y(\widetilde{U} \vec{k})$. If $y=\left(\mathcal{L}_{V} \searrow \mathcal{L}_{W}\right) x$ then:

$$
Y[\vec{k}]=\frac{1}{|\mathcal{J}(\mathrm{W}, V)|} \sum_{\vec{m} \in \mathcal{J}\left(Q^{T}\right)} X\left[\vec{k}+N^{T} \vec{m}\right]
$$

and if $y=\left(\mathcal{L}_{W} \nearrow \mathcal{L}_{V}\right) x$ then:

$$
Y[\vec{k}]=X\left[N^{T} \vec{k}\right]
$$

In order to avoid aliasing when decimating, we can first apply a filter $H$ to bandlimit the signal $x$ to a reciprocal unit cell $\mathcal{U}_{\widetilde{W}}$. This gives rise to a decimation filter operation:

$$
y=\left(\mathcal{L}_{V} \searrow \mathcal{L}_{W}\right) H x
$$

where the ideal filter has a spectrum $H(\vec{f})=\chi_{\mathcal{L}_{\widetilde{W}}}(\vec{f})$. This can be implemented by windowing (multiplying pointwise) in the frequency domain, or by convolution with the inverse transform $h$, which can be computed directly via the inverse MRL-DFT. The same ideal anti-aliasing filter also removes imaging distortion, i.e., retains only one copy of the spectrum when upsampling. Thus, the ideal interpolation filter is given by:

$$
y=H\left(\mathcal{L}_{W} \nearrow \mathcal{L}_{V}\right) x
$$

We can also specify ideal filtering operations for general sampling lattice conversion. Suppose $\mathcal{L}_{U} \subset \mathcal{L}_{W} \subset \mathcal{L}_{V}$ and $\mathcal{L}_{U} \subset \mathcal{L}_{P} \subset \mathcal{L}_{V}$ but the intervening lattices $\mathcal{L}_{W}, \mathcal{L}_{P}$ are not, in general a sublattice of the other. We want to convert a signal with support in $\mathcal{L}_{W}$ to one with support in $\mathcal{L}_{P}$, with ideal filtering to suppress both aliasing and imaging. In general, this may require some information loss (i.e., part of the original signal band may have to be suppressed). The ideal sampling rate conversion process is:

$$
y=\left(\mathcal{L}_{V} \searrow \mathcal{L}_{P}\right) H\left(\mathcal{L}_{W} \nearrow \mathcal{L}_{V}\right) x
$$

where the spectrum of $H$ is the indicator function for $\mathcal{U}_{\widetilde{W}} \cap \mathcal{U}_{\tilde{P}}$ (actually, points in $\mathcal{L}_{\widetilde{U}}$ that lie in this region).

Depending on the application, multirate filtering is not always designed to suppress aliasing or imaging, and in each of these configurations, the selected $H$ may be chosen based on different criteria. Additionally, it is recognized that the proposed schemes are not computationally efficient, though they are presented in forms that are directly related to the target application. In an interpolation filter, for example, a signal with many 0 values is fed to the filter, and thus many multiply operations are multiply by 0 , which is wasteful. Similarly, with a decimation filter, the filter is applied first, and then many computed values are discarded. The polyphase concept is useful in designing computationally efficient interpolation and decimation filtering algorithms, as well as in developing FFT algorithms.We will define polyphase components in our context below, both in the physical domain and in lexicographic form, and present one fundamental result that allows for efficient polyphase decomposition of filtering operations.

Let $\mathcal{L}_{U} \subset \mathcal{L}_{W} \subset \mathcal{L}_{V}$, and $x \in \mathcal{S}(U, V)$. Then each polyphase component of $x$ with respect to $\mathcal{L}_{W}$ is comprised of the values of $x$ on a single coset of $\mathcal{L}_{W}$ inside $\mathcal{L}_{V}$. With $W=V Q$, note that there are 
$|\operatorname{det} Q|$ distinct polyphase components. To be specific, each polyphase component is a signal in $\mathcal{S}(U, W)$, indexed by points in $\mathcal{L}_{V}$. If $\vec{k} \in \mathcal{L}_{V}$, then the corresponding polyphase component of $x$ is defined as:

$$
x_{\vec{k}}(\vec{r})=x(\vec{r}+\vec{k}) \text { for } \vec{r} \in \mathcal{L}_{W}
$$

Selecting the vectors $\vec{k}$ from any valid index $\operatorname{set} \mathcal{J}(W, V)$ generates all the distinct polyphase components.

Theorem 20: If $x, h$ have support in $\mathcal{L}_{V}$ (periodic or aperiodic), and $\mathcal{L}_{W} \subset \mathcal{L}_{V}$, then $y=h * x$ (i.e., either circular or linear convolution) can be computed in polyphase form as:

$$
y_{\vec{m}}=\sum_{\vec{k} \in \mathcal{J}(W, V)} h_{\vec{k}} * x_{\vec{m}-\vec{k}}
$$

Corollary 21: A decimation filter can be implemented efficiently via polyphase form as:

$$
\left(\mathcal{L}_{V} \searrow \mathcal{L}_{W}\right)(h * x)=\sum_{\vec{k} \in \mathcal{J}(W, V)} h_{\vec{k}} * x_{-\vec{k}}
$$

and an interpolation filter can be implemented efficiently via polyphase realization as follows:

$$
\left(h *\left(\left(\mathcal{L}_{W} \nearrow \mathcal{L}_{V}\right) x\right)\right)_{\vec{k}}=h_{\vec{k}} * x
$$

We note some final remarks on polyphase representation. The roles of space and frequency can be reversed, and we can define polyphase components by considering cosets of sublattices in the frequency domain. This is useful, for example, for regridding in the frequency domain from limited spectral measurements, or for implementing windowing operations (pointwise multiplication) in the spatial domain. Combining polyphase decomposition with prior results regarding the Fourier transform of signals with support on a coset of a lattice (Corollary 17) also leads to the development of FFT algorithms (i.e., generalizations of the standard decimation-intime and decimation-in-frequency formulations).

\section{RESULTS}

Here we discussan example to illustrate some important concepts that were developed in this paper. Start with the matrix $V$ given by:

$$
V=\left[\begin{array}{cc}
\sqrt{3} & \sqrt{3} \\
-1 & 1
\end{array}\right]
$$

Then $\mathcal{L}_{V}$ is generated by two vectors of equal length at an angle of $120^{\circ}$, and is called ahexagonal lattice [5]. We want to form multiresolution lattice chains $\mathcal{L}_{U} \subset \mathcal{L}_{W} \subset \mathcal{L}_{V}$ and $\mathcal{L}_{U} \subset \mathcal{L}_{P} \subset \mathcal{L}_{V}$ such that neither $\mathcal{L}_{W}$ nor $\mathcal{L}_{P}$ is a sublattice of the other.Then our goal is to describe the process of regridding, that is, for converting a signal sampled on $\mathcal{L}_{W}$ to one with support on $\mathcal{L}_{P}$. In particular, we want to determine the ideal interpolation filter.

Let us take $U=V M$ where $M=\operatorname{diag}\{8,8\}$. At first, chosing diagonal $M$ may seem simplistic, but recall that the Smith form of an integer matrix led to the result that we can always chose the generators of a lattice and sublattice so the matrices are related by a diagonal integer matrix. Now we can generate intervening lattices by (nontrivial) factorizations of $M$. Here, we will take $W=V N$ 
where $N=\operatorname{diag}\{2,1\}$, and $P=V Q$ where $Q=\operatorname{diag}\{1,2\}$. We can confirm that neither $\mathcal{L}_{W}$ nor $\mathcal{L}_{P}$ is a sublattice of the other by checking that neither $W^{-1} P$ nor $P^{-1} W$ is an integer matrix.

Figure 4 shows points in $\mathcal{L}_{W}$, marked ' $\mathrm{x}$ ', points in $\mathcal{L}_{P}$, marked ' + ', and points lying in both lattices marked ' $*$ '. Note that these common points themselves form a lattice $\mathcal{L}^{\prime}$ which is a sublattice of $\mathcal{L}_{W}$ and $\mathcal{L}_{P}$, and which contains $\mathcal{L}_{U}$ as a sublattice. The process for regridding has the form $\left(\mathcal{L}_{V} \searrow \mathcal{L}_{P}\right) H\left(\mathcal{L}_{W} \nearrow \mathcal{L}_{V}\right)$, which means first upsample to a dense lattice, apply an interpolation filter $H$ which in this case combats aliasing and imaging distortion, and downsample to the target lattice. In the frequency domain $H$ has support on the reciprocal lattice $\mathcal{L}_{\widetilde{U}}$ and is in fact 1 at points inside $\mathcal{U}_{\widetilde{W}} \cap \mathcal{U}_{\tilde{P}}$, and 0 outside, for some choice of unit cells. Here, we take the unit cell for a lattice generated by $A$ to be $A\left[-\frac{1}{2}, \frac{1}{2}\right)^{D}$. We can check if a point $\vec{\zeta}$ is inside such a set by computing $A^{-1} \vec{\zeta}$. For this example, Figure 5 shows the frequency points $\vec{f} \in \mathcal{L}_{\widetilde{U}}$ and highlights the points where ideal $H(\vec{f})=1$ with filled circles.

We can perform regridding of a signal $x$ from $\mathcal{L}_{W}$ to $\mathcal{L}_{P}$ by working in the frequency domain:

1. Compute the $\mathcal{L}_{W} / \mathcal{L}_{U}$ MRL-DFT of $x$.

2. Multiply pointwise by $H(\vec{f})$, which in this case just means retain the spectral coefficients that lie within $\mathcal{U}_{\widetilde{W}} \cap \mathcal{U}_{\tilde{P}}$, and discard (i.e., set to 0 ) the rest.

3. Compute the $\mathcal{L}_{P} / \mathcal{L}_{U}$ inverse MRL-DFT.

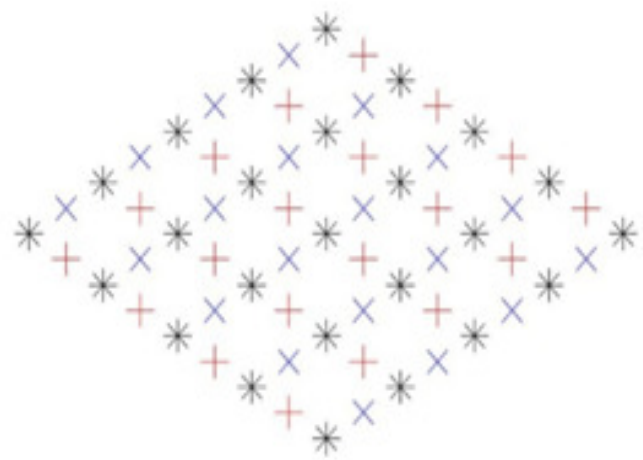

Figure 4: Two lattices, marked ' $x$ ' and ' + ' respectively, with common points marked '*'. We wish to convert a signal sampled on the ' $x$ ' lattice to one with support on the ' + ' lattice.

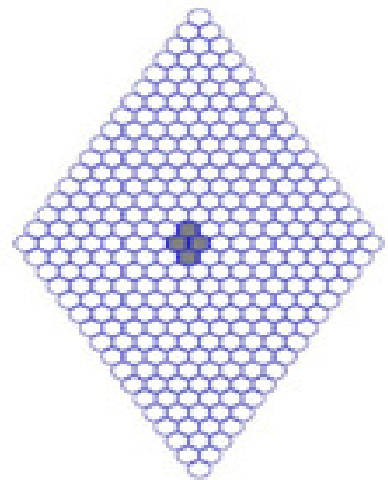

Figure 5: The passband of an ideal interpolation filter, as seen on the reciprocal lattice. 
The operation can also be performed in the spatial domain. For this, we need to convolve with the impulse response $h(\vec{r})$ of $H$, which has support on $\mathcal{L}_{V}$. The coefficients $h$ (which are complex valued, in general) can be found by computing the $\mathcal{L}_{V} / \mathcal{L}_{U}$ inverse MRL-DFT of $H$. The result for this case is shown in Figure 6. In order to illustrate the filter coefficients, we show a contour plot of $|h(\vec{r})|$ if it were computed on the continuum $\vec{r} \in \mathcal{R}^{D}$ and then mark with ' $\times$ ' the points on $\mathcal{L}_{V}$ where it should be sampled. The filter coefficients satisfy symmetry conditionsh $(x, y)=$ $h(x,-y)=h^{*}(-x,-y)$ and the values at points in the first quadrant are given in Table 1.

Table 1: Computed ideal regridding coefficients.

\begin{tabular}{|c|c|c|}
\hline$\vec{r}$ & $\overrightarrow{\boldsymbol{n}}$ & $\boldsymbol{h}(\overrightarrow{\boldsymbol{r}})$ \\
\hline$(0,4)$ & $(-2,2)$ & $2+j 0$ \\
\hline$(\sqrt{3}, 5)$ & $(-2,3)$ & $1+j(-\sqrt{2}+1)$ \\
\hline$(0,2)$ & $(-1,1)$ & $(\sqrt{2}+2)+j 0$ \\
\hline$(\sqrt{3}, 3)$ & $(-1,2)$ & $(\sqrt{2}+1)-j 1$ \\
\hline$(2 \sqrt{3}, 4)$ & $(-1,3)$ & $1-j 1$ \\
\hline$(0,0)$ & $(0,0)$ & $2+j 0$ \\
\hline$(\sqrt{3}, 1)$ & $(0,1)$ & $(\sqrt{2}+2)-j \sqrt{2}$ \\
\hline$(2 \sqrt{3}, 2)$ & $(0,2)$ & $2-j 2$ \\
\hline$(3 \sqrt{3}, 3)$ & $(0,3)$ & $(2-\sqrt{2})-j \sqrt{2}$ \\
\hline$(2 \sqrt{3}, 0)$ & $(1,1)$ & $1-j(\sqrt{2}+1)$ \\
\hline$(3 \sqrt{3}, 1)$ & $(1,2)$ & $0-j \sqrt{2}$ \\
\hline$(4 \sqrt{3}, 2)$ & $(1,3)$ & $0-j 2$ \\
\hline$(4 \sqrt{3}, 0)$ & $(2,2)$ & $(-\sqrt{2}+1)-j 1$ \\
\hline$(5 \sqrt{3}, 1)$ & $(2,3)$ & $(-\sqrt{2}+1)+j(-\sqrt{2}+1)$ \\
\hline$(6 \sqrt{3}, 0)$ & $(3,3)$ & \\
\hline
\end{tabular}

Then, conceptually, the spatial domain regridding algorithm is:

1. Upsamplex from $\mathcal{L}_{W}$ to $\mathcal{L}_{V}$ by inserting 0 's.

2. Interpolate the values by convolving with $h$.

3. Decimate down to $\mathcal{L}_{P}$.

This process, in reality, would be realized in polyphase form to achieve computational efficiency, as described in this paper.

Figure 6: Ideal regridding filter coefficients, located at the lattice points marked 'x', with an indicated contour plot of their amplitude. 
Observe that the process of identifying sublattices, and developing multirate algorithms, such as regridding, is relatively straightforward because the physical nature of the spatial and frequency domain coordinates is retained. Directly finding the lexicographic indices of the support of the interpolation filter, for example, would be much more challenging than the approach taken here.

\section{FUTURE WORK}

The MRL-DFT and related results presented in this paper form the foundation for the derivation of a number of important signal processing algorithms. One area to be studied is the formulation of FFT algorithms for multiresolution lattices. The results presented here on spectral properties of signals with support on cosets of sublattices could potentially be used to generate general treestructured FFT algorithms for multiresolution lattices, in which spectral information of multiresolution signals can be studied directly, without conversion to a universal (high density) resolution. Another topic for further inquiry is formulating fast algorithms for regridding (interpolation), based on generalization of fast convolution operations known for 1-D (and separable multidimensional) cases.

\section{Conclusions}

We have presented a framework for processing signals on general, variable density sampling grids in the spatial and frequency domains. We formulated the signal processing operations in terms of physically meaningful spatial and frequency coordinates, which retains the significance of spatial correlations in the signal. The underlying mathematical approach was to consider lattice chains, and develop the MRL-DFT as the finite Fourier transform over associated quotient groups, This led to formulation of important relationships, such as the convolution theorem, aliasing and imaging associated with multirate operations, and development of interpolation (regridding) algorithms. We also presented techniques for analysing the lattice structures, for example construction of multiresolution sublattices. Special properties, such as the spectrum associated with signals restricted to sublatticecosets, and the polyphase form, can lead to the further development of special algorithms such as generalized FFT. We related our results to the standard lexicographic form that uses normalized integer indices. In the end, data is stored and processed in rectangular arrays, as computer memory has that structure, but algorithms are developed and understood better in a physical coordinate system. The techniques presented here are useful in a myriad of imaging applications where data is obtained in either the spatial or frequency domains on a non-rectangular grid or possibly an irregular point set with potentially variable density. For example, rather than being forced to artificially regrid data collected at variable densities to a grid with uniform density, the interpolation can be targeted for a multiresolution lattice structure, which can be processed directly. This can help avoid artifacts caused by regridding to densities that are locally too high or too low compared to that of the original data.

\section{REFERENCES}

[1] Dudgeon, D. \&Mersereau, R. (1983).Multidimensional digital signal processing.Saddle Brook, NJ: Prentice-Hall.

[2] Xing, S. \& Li, Y. \& Wang, X. (2013). Three-dimensional reconstruction of man-made objects using polarimetric tomographic SAR.IEEE Trans. Geosci.\& Rem. Sens., 51(6), 3694-3705.doi: 10.1109/TGRS.2012.2220145.

[3] Vaidyanthan, P.P. (1993).Multiratesystems \&filter banks. Saddle Brook, NJ:Prentice-Hall. 
[4] Van de Walle, R. \&Lemahieu, I. (2000). Reconstruction of MR images from data acquired on a general nonregular grid by pseudoinverse calculation.IEEE Trans. Med. Imag., 19(12), 1160-1167. doi: S 0278-006200)10881-X.

[5] Conroy, B. (2005). Reconstruction of spiral MRI using FFT on hexagonal lattice structures (Master's Thesis). Retrieved from Cooper Union Archives. (C 7A2 EE 2005 C754).

[6] Yang, Y. \& Liu, Q. (2017). Pseudo-polar Fourier transform-based compressed sensing MRI. IEEE Trans. Biomed. Eng., 64(4), 816-826.doi: 10.1109/TBME.2016.2578930.

[7] He, X.Y. \& Zhou, X.Y. (2012). Fast 3D-ISAR image simulation of targets at arbitrary aspect angles through nonuniform fast Fourier transform (NUFFT).IEEE Trans. Ant. \&Propag., 60(5), 25972602.doi: 10.1109/TAP.2012.2189717.

[8] Rundblad, E. \&Astola, J. \&Egiazarian, K. (2002). Fast algorithms of multidimensional discrete nonseparable K-wave transforms.IEEE Trans. Sig. Proc., 50(6), 1496-1507.doi: S1053$587 \mathrm{X}(02) 04400-8$.

[9] Moustakas, A. \&Mertikopoulos, P. \&Bambos, N. (2016). Power optimization in random wireless networks.IEEE Trans.Info. Theory, 62(9), 5030-5058.doi: 10.1109/TIT.2016.2594183.

[10] Ezzeldin, Y.\&Seddik, K. (2016). Pseudo-lattice treatment for subspace aligned interference signals.IEEE Trans. Vehic. Tech., 63(9), 4728-4734.doi: 10.1109/TVT.2014.2317753.

[11] Peng, S. \&Liu, A. \& Wang, H. (2017). Hexagonal multicarrier faster-than-Nyquist sampling. IEEE Access, 5(1), 3322-3339.doi: 10.1109/ACCESS.2017.2674666.

[12] Huang, Y.C. (2017). Lattice index codes from algebraic number fields.IEEE Trans. Info. Theory, 63(4), 2098-2112.doi: 10.1109/TIT.2017.2655034.

[13] Zhang, W.\& Wang, W. (2016). Signal shaping and precoding for MIMO systems using lattice codes.IEEE Trans. Wireless Comm., 15(7), 4625-4634.doi: 10.1109/TWC.2016.2543213.

[14] Muramatsu, S. \&Furuya, K.\&Yuki, N. (2017). Multidimensional nonseparableoversampled lapped transforms: theory and design.IEEE Trans. Sig. Proc., 65(5), 1251-1264. doi: 10.1109/TSP.2016.2633240.

[15] Rauh, A. \&Arce, G. (2017). Optimized spectrum permutation for the multidimensional sparse FFT.IEEE Trans. Sig. Proc., 65(1), 162-172.doi: 10.1109/TSP.2016.2599483.

[16] Horn, R.\& Johnson, C. (2012).Matrix Analysis. Cambridge, UK:Cambridge Press.

\section{AUTHOR}

Fred L. Fontaine is the Jesse Sherman Professor and chair of the Department of Electrical Engineering at The Cooper Union in New York City. He has a Ph.D. (Stevens Inst.), M.E. (Cooper Union), and B.E. (Cooper Union) in electrical engineering, and an M.S. (Courant Inst. NYU) in math. He has extensive experience as a research consultant in industry. His areas of interest include multidimensional and multi rate signal processing, radar, communication systems, probability and stochastic processes, machine learning and processor architectures. He is a member of Tau Beta Pi, Eta Kappa Nu and IEEE.

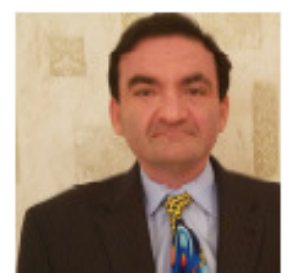

